\title{
Impacto del Ácido Fólico en la Incidencia de Fisuras Labiopalatinas de la Población Occidente de la Región Metropolitana, Chile
}

\author{
Impact of Folic Acid on the Incidence of Cleft Lip and Palate \\ of the Western Population of the Metropolitan Region, Chile
}

Camila Jara P.'; Rodrigo Soto R. ${ }^{1}$; Consuelo Tello T. ${ }^{1}$ \& Benjamín Martínez R. ${ }^{1,2}$

JARA, P. C.; SOTO, R. R; TELLO, T. C. \& MARTíNEZ, R. B. Impacto del ácido fólico en la incidencia de fisuras labiopalatinas de la población occidente de la Región Metropolitana, Chile. Int. J. Odontostomat., 12(3):228-232, 2018.

RESUMEN: La fisura labiopalatina ha tenido una alta frecuencia en la población chilena. A partir del 2000 se inició el programa de fortificación de la harina con ácido fólico a fin de disminuir los defectos del tubo neural. El objetivo de este estudio fue determinar el impacto que presenta la incorporación del ácido fólico en la incidencia de fisuras labiopalatinas de la población del área occidente de la Región Metropolitana. Se recopiló información a partir de las fichas clínicas de la Fundación Gantz seleccionando a los recién nacidos entre 1990 y 2009, correspondientes a las comunas del Servicio de Salud Occidente de la Región Metropolitana. Desde el 2000 hasta el 2004 se observó una disminución importante en las tasas de fisura labiopalatina, con una tasa de 0,88 fisurados por 1000 recién nacidos vivos. Posteriormente se observó un incremento a partir del 2005 hasta el 2009 llegando a una tasa de 1,52 fisurados por 1000 recién nacidos vivos. En conclusión, el efecto protector del ácido fólico se evidencia en la disminución de las tasas de los recién nacidos con fisuras durante los primeros años de su implementación. Sin embargo, posteriormente se observó un aumento de dichas tasas, lo cual podría deberse al incumplimiento de la norma.

PALABRAS CLAVE: labio fisurado, paladar fisurado, ácido fólico, alimentos fortificados.

\section{INTRODUCCIÓN}

Las malformaciones congénitas son anomalías del desarrollo embrionario, que están presentes al momento del nacimiento (Nazer, 2004) Estas constituyen la causa principal de mortalidad infantil y pueden ser estructurales, funcionales, metabólicas, conductuales o hereditarias (Moore, 2008).

Dentro de las malformaciones congénitas se encuentran las fisuras cráneo-faciales, que a nivel mundial describen una prevalencia de 7,94 por cada 10.000 Recién Nacidos Vivos (RNV) (Tanaka et al., 2012). La fisura labial con o sin paladar fisurado es una de las malformaciones más comunes en el ser humano y la más frecuente de cabeza y cuello (Antoszewski \& Fija kowska, 2016).

Estudios de la Universidad de Chile, el Estudio Colaborativo Latino Americano de Malformaciones
Congénitas y la Fundación Gantz señalan 1,8 casos por cada 1000 RNV en Chile (1 por cada 585 RNV); no obstante, hay diferencias geográficas que muestran mayor incidencia en las regiones de Tarapacá, Antofagasta y Araucanía (Fundación Gantz, 2016).

Se sabe que la fisura labiopalatina es una malformación congénita compleja y heterogénea y su ocurrencia se asocia con varios factores de riesgo tanto ambientales como genéticos, con probables interacciones entre ellos (Jia et al., 2011; Kelly et al., 2012).

Los defectos del tubo neural y las fisuras orales están relacionados embriológicamente. Los tejidos faciales y dentarios se desarrollan a partir de las células de la cresta neural que se originan del proceso dorso lateral del tubo neural en desarrollo. Por lo tanto, se ha planteado la hipótesis de que el ácido fólico

\footnotetext{
${ }^{1}$ Cirujano Dentista, Facultad de Odontología, Universidad Mayor, Santiago, Chile.

${ }^{2}$ Patólogo Oral y Maxilofacial, Facultad de Odontología, Universidad Mayor, Santiago, Chile.
} 
periconcepcional, también podría proteger contra las anomalías congénitas comunes como la fisura labiopalatina (Badovinac et al., 2007).

Se aconseja a las mujeres en edad fértil tomar un adicional de $400 \mathrm{mg}$ de ácido fólico todos los días si hay alguna posibilidad de quedar embarazada. El cierre del tubo neural se completa al final de la quinta semana de gestación, momento en el cual la mujer, por lo general, recién está teniendo conciencia de su embarazo. Es por esto que toda medida preventiva debe ser desde al menos dos meses antes de la concepción y durante los dos primeros meses de gestación (Cortés et al., 2000; Lardoeyt Ferrer et al., 2005).

En la práctica, el consumo de ácido fólico preventivo ha tenido captación mixta. Sin embargo, se advierte que los embarazos no planificados pueden dificultar el éxito en la reducción de este problema. Por ello, en algunos países se ha introducido la fortificación obligatoria con ácido fólico de los alimentos debido a los beneficios para la salud de la población (Cortés et al., 2000; Lardoeyt Ferrer et al.).

En Chile, las autoridades de salud basadas en todos los antecedentes disponibles determinaron que a partir del 1 de enero del 2000 era obligatoria la fortificación de la harina de panificación (Resolución Exenta $\mathrm{N}^{\circ} 543$ del 2000). El nivel de fortificación elegido es de $220 \mu \mathrm{g}$ de ácido fólico por cada $100 \mathrm{~g}$ de harina. Con esta medida se pretende alcanzar un aporte mínimo de $360 \mu \mathrm{g}$ diarios de ácido fólico para la mujer en edad fértil sólo a través del consumo de pan fortificado, dado que el promedio de ingesta de pan es de 220 g diarios (Cortés et al., 2000).

A partir de esta información, es interesante observar si la fortificación de la harina con ácido fólico ha tenido impacto en la incidencia de la fisura labiopalatina, por lo que el objetivo de este estudio es determinar la influencia que presenta la incorporación del ácido fólico en la presencia de fisuras en la población del área occidente de la Región Metropolitana antes y después de la puesta en marcha de esta política pública.

\section{MATERIAL Y MÉTODO}

Se realizó un estudio analítico retrospectivo, en el que se observó el impacto que presentó la fortificación de ácido fólico en la incidencia de fisura labiopalatina en el área occidente de la Región Metropolitana.
Se determinaron como fuente de recolección de información los Servicios de Maternidad y Neonatología de los cinco hospitales correspondientes al Servicio de Salud Metropolitano Occidente de la Región Metropolitana, a saber: Hospital San Juan de Dios, Félix Bulnes, Talagante, Peñaflor y Melipilla (considerando que el $70 \%$ de los partos en Chile se atienden en el sector público de salud) (Hertrampf \& Cortés, 2004).

Se informó que, durante el periodo de recolección de datos (1990-2009), todos los niños nacidos con fisura labiopalatina en los hospitales correspondientes al Servicio de Salud Occidente eran derivados a la Fundación Gantz, institución chilena dedicada al tratamiento y la rehabilitación integral de los niños portadores de fisuras faciales. Por esta razón, los datos necesarios se obtuvieron a partir del análisis de las fichas clínicas de los niños atendidos entre 1990 y 2009 derivados desde los hospitales del Servicio Occidente de la Región Metropolitana a este organismo.

Los datos fueron analizados estadísticamente mediante análisis de regresión lineal y correlación de Pearson, con el fin de determinar la asociación existente entre la cantidad de fisuras y la incidencia entre 1990 y 2009. Los datos fueron procesados y graficados por el software estadístico Stata v 13.1. Para resguardar la ética del estudio, solo se trabajó con datos estadísticos y no se usó información confidencial de cada paciente y sus padres.

Para analizar la fortificación de la harina con ácido fólico, se recopilaron datos desde el Instituto de Salud Pública (ISP) que se encontraban tabulados en planillas Excel. Estos datos incluían muestras de distintos molinos de todo Chile elegidos al azar por dicha institución desde el 2005 al 2009, los cuales fueron analizados para determinar el cumplimiento de la norma de fortificación de ácido fólico. Posteriormente, estos datos fueron procesados en Excel para calcular los porcentajes de muestras que se encontraban por debajo de la norma.

\section{RESULTADOS}

En el registro del total de Recién Nacidos Vivos del período de enero de 1990 a diciembre de 2009 en los hospitales correspondientes a la zona occidente de la Región Metropolitana se observaron 416 niños con fisura de un total de 307.901 RNV. 
Se analizaron las tasas brutas de fisuras incluyendo el total de niños nacidos con fisura labial, fisura labial y palatina, y fisura palatina, entre 1990 y 2009 (Fig. 1) y se constata que hubo un aumento creciente hasta 1999, con una tasa de 1,85 fisurados por cada 1000 RNV. Desde el 2000 se observó una disminución importante en las tasas hasta el 2004, con una tasa de 0,88 fisurados por cada 1000 RNV. Sin embargo, posteriormente se registró un incremento a partir del 2005 hasta el 2009, con una tasa de 1,52 fisurados por cada 1000 RNV, similar a las tasas obtenidas a principios de la década del 90.

Se analizaron las tasas brutas desde 1990 hasta 2004 y se observó una disminución progresiva del número de fisuras labiopalatinas a partir del 2000, con una tasa de 0.88 por cada 1000 recién nacidos vivos el 2004 (Fig. 2), es decir, la tasa de menor valor del período de estudio. Al analizar estos datos estadísticamente se obtuvo correlación de Pearson $r=0,745(y=-0,0115 \times 2+$ $45,926 x-45838$, y R2 de 0,5561, es decir, $55,61 \%$ de la variación de las fisuras labiopalatinas podría explicarse en este modelo según los cambios entre 1990 y 2004.

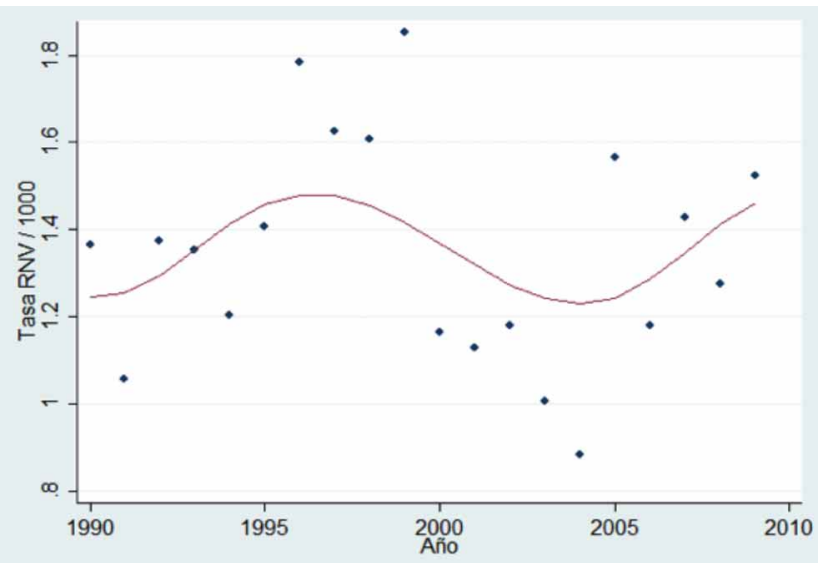

Fig. 1. Tasas brutas de fisurados x 1.000 R.N.V del Servicio Occidente Región Metropolitana, Chile desde 1990 a 2009.

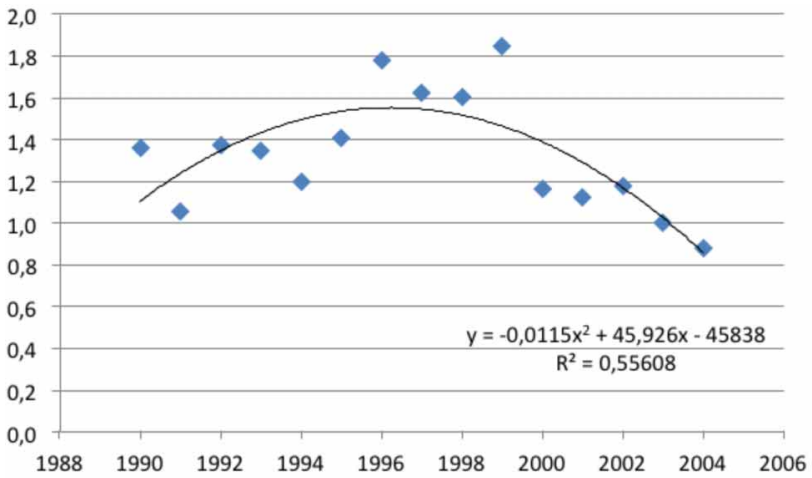

Fig. 2. Tasas brutas de fisurados $\times 1.000$ R.N.V del Servicio Occidente Región Metropolitana, Chile desde 1990 a 2004.

\section{DISCUSIÓN}

Múltiples estudios de intervención y de observación realizados durante los últimos 25 años han permitido determinar que el ácido fólico consumido periconcepcionalmente ejerce un rol protector sobre los defectos del cierre del tubo neural (DTN) (Wilson et al., 2003), pero aún no está completamente definido su efecto sobre las fisuras labiopalatinas.

Del análisis de los resultados obtenidos en este estudio en relación a las tasas de recién nacidos fisurados por año, se pudo observar una importante disminución a partir del 2000. Al compararla con la tasa de 1999, se evidencia una reducción en más de la mitad. Sin embargo, a partir del 2005 se registró un aumento considerable en el número de fisurados, con una tasa similar a la encontrada en los años previos a la fortificación de la harina. Por lo tanto, si bien durante los primeros años se observó una reducción en el número de fisuras labiopalatinas atribuibles a la fortificación de ácido fólico en la harina, las variaciones posteriores en las tasas permiten establecer que, en este estudio, el rol protector que presenta el ácido fólico no es concluyente.

Entre las posibles explicaciones del aumento de las fisuras labiopalatinas en el periodo de 2005 a 2009, se pueden citar:

1. En los últimos 20 años el pan ha ido perdiendo participación en la dieta de los chilenos, pasando de 98 a 86 kilos per cápita al año (2010). Según un estudio de Fechipan, su consumo ha sido en promedio de 85,6 kilos por persona al año, entre 2001 y 2010. Dentro del estudio se indica que el pro-ducto ha tenido una variación entre 1928 y 2008 de $8,9 \%$ a $2 \%$ en la participación de la canasta del IPC. En otras palabras, ha bajado su consumo en los hogares (ODEPA, 2012).

2. También es posible que la forma de administración de ácido fólico no sea la óptima. El $85 \%$ del ácido fólico de los alimentos fortificados es absorbido por el organismo, a diferencia del consumo de suplementos vitamínicos, de los cuales se absorbe el $100 \%$ (Lindzon \& O'Connor, 2007).

3. Según datos obtenidos del ISP, el $63,71 \%$ de las fábricas de harina presentaron una subfortificación de ácido fólico con respecto a la norma (fortificación de las harinas con ácido fólico entre 2,0 a $2,4 \mathrm{mg} / \mathrm{Kg}$ ) entre el 2005 y el 2009 (Tabla I). Lamentablemente 
Tabla I. Porcentaje de Molinos que presentan subfortificación de Ácido Fólico (2005 - 2009).

$\begin{array}{lr} & \text { No Cumplen con la norma } \\ 2005 & 55,43 \% \\ 2006 & 59,08 \% \\ 2007 & 62,95 \% \\ 2008 & 75,82 \% \\ 2009 & 65,28 \% \\ \text { Promedio } & 63,71 \%\end{array}$

los registros entre el 2000 y 2004 no se encontraban disponibles, ya que fueron eliminados de dicha institución. Los únicos datos registrados sobre la fortificación de este período son de un estudio de Hertramp \& Cortés (2004), en el que se evaluó el contenido de folato en el pan de las panaderías de Santiago un año tras la implementación de la norma. En este estudio se observó un incumplimiento de solo el $26 \%$ de la fortificación. Por lo tanto, pareciera que el cumplimiento de la norma de ácido fólico incorporado en la harina durante los primeros años era mayor que los años posteriores al 2004. Esto podría estar afectando el incremento de niños fisurados y otras alteraciones del tubo neural.

Según el estudio realizado por Cortés et al. (2012), en el que se analizó la frecuencia de defectos del tubo neural en recién nacidos vivos de nueve hospitales de Santiago, durante 1990 al 2009, se pudo observar disminución tras la fortificación de las harinas con ácido fólico y posterior aumento a partir del año 2004. Esto concuerda con los resultados obtenidos en el presente estudio.

Debido a lo anteriormente expuesto, es que se estima que una de las razones más relevantes por la cual se ha registrado un aumento en la incidencia de las fisuras labiopalatinas entre 2005 y 2009 , sería el no cumplimiento de la norma establecida en el 2000 (Resolución Exenta N 543 del 2000) por la mayoría de los molinos del país.

Se sugiere implementar una mayor fiscalización por parte de las autoridades para el cumplimiento de la cantidad establecida de ácido fólico incorporada en la harina, debido a que las fisuras labiopalatinas son un problema de salud pública, al igual que los defectos del tubo neural, y el tratamiento de un paciente que presente esta patología es de mayor costo para el país que el de la fortificación de la harina con ácido fólico. Todo esto sumado al daño psicológico causado al niño y su familia.
Además de lo anterior, se sugiere realizar nuevos estudios para analizar si desde el 2010 al presente, han ocurrido cambios en el cumplimiento de la norma de fortificación de las harinas con ácido fólico, y si continúa el aumento en las tasas de incidencia de fisuras labiopalatinas en Chile.

JARA, P. C.; SOTO, R. R; TELLO, T. C. \& MARTÍNEZ, R. B. Impact of folic acid on the incidence of cleft lip and palate of the western population of the Metropolitan Region, Chile. Int. J. Odontostomat., 12(3):228-232, 2018.

ABSTRACT: Cleft lip and palate has had a high frequency in the Chilean population. From the year 2000 a flour fortification program with folic acid was initiated in order to reduce the defects of the neural tube. The objective of this study was to determine the impact of the incorporation of folic acid in the incidence of cleft lip and palate of the population of the western area of the Metropolitan Region. Information was collected from clinical records of the Gantz Foundation, selecting newborns from 1990 to 2009, corresponding to the districts of the western sector of the Metropolitan Region. From 2000 to 2004 there was a significant decrease in cleft lip and palate rates, with a rate of 0.88 cases per 1000 live births. Subsequently, an increase was observed from 2005 to 2009 , reaching a rate of 1.52 cases per 1000 live births. In conclusion, the protective effect of folic acid is evidenced in the reduction of the rates of newborns with cleft palate during the first years of its implementation. However, an increase in these rates was subsequently observed, which could be due to noncompliance with the standard.

KEY WORDS: cleft lip, cleft palate, folic acid, fortified foods.

\section{REFERENCIAS BIBLIOGRÁFICAS}

Antoszewski, B. \& Fijakowska, M. Prevalence of cleft lip and/or palate in children from Lodz between years 1981-2010. Congenit. Anom. (Kyoto), 56(2):60-4, 2016.

Badovinac, R. L.; Werler, M. M.; Williams, P. L.; Kelsey, K. T. \& Hayes, C. Folic acid-containing supplement consumption during pregnancy and risk for oral clefts: a meta-analysis. Birth Defects Res. A Clin. Mol. Teratol., 79(1):8-15, 2007.

Cortés, F.; Mellado, C.; Pardo, R. A.; Villarroel, L. A. \& Hertrampf, E. Wheat flour fortification with folic acid: changes in neural tube defects rates in Chile. Am. J. Med. Genet. A, 158A(8):1885-90, 2012.

Cortés, M. F.; Hirsch, B. S. \& de la Maza, C. M. P. Importancia del ácido fólico en la medicina actual. Rev. Med. Chile, 128(2):21320, 2000.

Fundación Gantz. Incidencias. Santiago, Fundación Gantz, 2016. Disponible en: https://gantz.cl/2016/04/todo-por-el-nino-fisurado/

Hertrampf, E. \& Cortés, F. Folic acid fortification of wheat flour: Chile. Nutr. Rev., 62(6 Pt. 2):S44-8, 2004.

Jia, Z. L.; Shi, B.; Chen, C. H.; Shi, J. Y.; Wu, J. \& Xu, X. Maternal malnutrition, environmental exposure during pregnancy and the 
JARA, P. C.; SOTO, R. R; TELLO, T. C. \& MARTíNEZ, R. B. Impacto del ácido fólico en la incidencia de fisuras labiopalatinas de la población occidente de la Región Metropolitana, Chile. Int. J. Odontostomat., 12(3):228-232, 2018.

risk of non-syndromic orofacial clefts. Oral Dis., 17(6):584-9, 2011. Kelly, D.; O'Dowd, T. \& Reulbach, U. Use of folic acid supplements and risk of cleft lip and palate in infants: a population-based cohort study. Br. J. Gen. Pract., 62(600):e466-72, 2012.

Lardoeyt Ferrer, R.; Taboada Lugo, N.; Torres Sánchez, Y. \& Viñas Portilla, C. Fundamentos del ácido fólico en la prevención primaria farmacológica de defectos congénitos. Rev. Cuba. Med. Gen. Integral, 21(1-2), 2005

Lindzon, G. \& O'Connor, D. L. Folate during reproduction: the Canadian experience with folic acid fortification. Nutr. Res. Pract., 1(3):163-74, 2007.

Moore, K. Embriología Clínica. Madrid, Elsevier, 2008. pp.457-86.

Nazer, H. J. Prevención primaria de los defectos congénitos. Rev. Med. Chile, 132(4):501-8, 2004.

Oficina de Estudios y Políticas Agrarias (ODEPA). Consumo Aparente de Principales Alimentos en Chile. Santiago de Chile, ODEPA Ministerio de Agricultura, 2012. Disponible en: http:// www.odepa.gob.cl/odepaweb/publicaciones/doc/7004.pdf

Tanaka, S. A.; Mahabir, R. C.; Jupiter, D. C. \& Menezes, J. M. Updating the epidemiology of cleft lip with or without cleft palate. Plast. Reconstr. Surg., 129(3):511e-8e, 2012.

Wilson, R. D.; Davies, G.; Désilets, V.; Reid, G. J.; Summers, A.; Wyatt. P.; Young, D. \& Genetics Committee and Executive and Council of the Society of Obstetricians and Gynaecologists of Canada. The use of folic acid for the prevention of neural tube defects and other congenital anomalies. J. Obstet. Gynaecol. Can., 25(11):959-73, 2003.
Dirección para correspondencia:

Dra. Camila Jara Pacheco

Fernando Lazcano 1280, Departamento 1602

Santiago

CHILE

\section{Email: Camilajarapacheco@gmail.com}

Recibido : 22-02-2018

Aceptado: 31-05-2018 